\title{
Procedencia de los sílex recuperados en Praileaitz I (Deba, Gipuzkoa)
}

\author{
Praileaitz I (Deba, Gipuzkoa) haitzuloan berreskuratutako suharrien jatorria \\ Provenance of recovered flint in Praileitz I (Deba, Gipuzkoa)
}

PALABRAS CLAVE: Materias Primas, Sílex, Flysch, Treviño, Urbasa, Chalosse.

GAKO-HITZAK: Lehengaia, Suharria, Flysch, Trebiñu, Urbasa, Chalosse. KEYWORDS: Raw Material, Flint, Flysch, Treviño, Urbasa, Chalosse.

Antonio TARRIÑO VINAGRE(1)

\section{RESUMEN}

Se ha revisado la práctica totalidad de los restos líticos recuperados en el yacimiento. Se trata de un conjunto bastante bien conservado y homogéneo. Se han identificado seis tipos de sílex diferentes: sílex del Flysch con un 80,5\% como sílex mayoritario; tres tipos minoritarios como los sílex de Treviño (3,7\%), Urbasa $(2,6 \%)$ y Chalosse $(2,1 \%)$ y otros dos tipos con una aparición anecdótica como los de sílex nordpirenaicos de Tercis $(0,8 \%)$ y Salies de Béarn $(0,3 \%)$. La aparición de sílex minoritarios está muy homogéneamente repartida entre los distintos niveles.

\section{LABURPENA}

Aztarnategian berreskuratutako harrizko aztarna ia guztiak aztertu dira. Bilduma ondo kontserbatzen da eta homogeneoa da. Sei suharri- edo silex-mota ezberdin identifikatu dira: Flyschekoa da suharri gehiengoa \%80,5arekin; gutxiengo beste hiru, Trebiñukoa \% 3,7arekin, Urbasakoa \% 2,6arekin eta Chalossekoa \% 2,1arekin, eta ohikoak ez diren beste bi mota Pirinioen iparraldekoak, Tercisekoa \% 0,8arekin eta Saliese-koa \% 0,3arekin. Gutxiengoa diren suharriak homogeneoki banatuta agertzen dira maila guztietan.

\section{SUMMARY}

It has been revised almost the entire lithic remains recovered at the site. This is an ensemble well preserved and homogeneous. Six different types have been identified: Flysch with $80.5 \%$ as major flint; three minor types: Treviño (3.7\%), Urbasa (2.6\%) and Chalosse (2.1\%); and two more with a superficial presence, which correspond to north-pyrenean varieties: Tercis $(0.8 \%)$ and Salies de Béarn $(0.3 \%)$. The supply of minor flints is evenly distributed among the levels.

\section{1.- INTRODUCCIÓN}

La cueva de Praileaitz I (Deba -Gipuzkoa-) se sitúa a unos tres kilómetros del mar sobre calizas arrecifales del Complejo Urgoniano (Cretácico inferior). Este sector de la cuenca del bajo Deba está profundamente karstificado, conociéndose varios yacimientos de cronología parcialmente paralelizable con la cavidad que tratamos. Entre estas destacamos Ermittia, Iruroin, Langatxo, Aizkoltxo, Agarre, etc.

Con el objeto de determinar la procedencia de los sílex de Praileaitz I se ha revisado la práctica totalidad de los restos recuperados en los distintos sectores del yacimiento (vestíbulo, tránsito entre el vestíbulo y la primera sala interior y primera sala interior). No han sido estudiados los del rellano exterior, la galería noroeste y la segunda sala interior.

La cronología de los diferentes niveles, sintéticamente, es la siguiente: I. Nivel superficial -prácticamente es- téril-; II. Epipaleolítico; III. Magdaleniense Superior-Final; IV. Magdaleniense Inferior y V. Solutrense.

El método de trabajo empleado ha sido el análisis textural de los sílex. Las determinaciones se han realizado a nivel macroscópico utilizando una lupa binocular convencional con aumentos comprendidos entre 6,0 y 45,0 aumentos.

Se trata de una colección que en general se encuentra bien conservada. Solo en 12 casos (3,1\%) se aprecia que los sílex están afectados por fuerte patinación con pérdida de masa y en un solo caso $(0,3 \%)$ se ha detectado una patinación grisácea originada por haber estado en contacto directo con el fuego.

En cuanto al grado de fracturación podemos afirmar que se trata de una colección bastante fracturada. Se han identificado 245 fragmentos que están rotos o son trozos informes, que suponen un $63 \%$ del conjunto

\footnotetext{
(1) Centro Nacional de Investigación sobre la Evolución Humana (CENIEH). Paseo Sierra de Atapuerca 3, 09002 Burgos. antonio.tarrinno@
} cenieh.es 
(prácticamente los dos tercios). El grado de fracturación afecta de un modo uniforme a todo el conjunto con diferencias muy poco significativas en los diferentes niveles (Tabl.1).

\begin{tabular}{|c|c|c|c|}
\hline Nivel & Fracturados & $\begin{array}{c}\text { Índice de } \\
\text { fracturación }\end{array}$ & Cronología \\
\hline II & $91 / 144$ & 0,63 & Epipaleolítico \\
\hline III & $63 / 96$ & 0,66 & Magdaleniense Superior \\
\hline IV & $67 / 110$ & 0,61 & Magdaleniense Inferior \\
\hline V & $17 / 30$ & 0,57 & Solutrense \\
\hline TOTAL & $238 / 380$ & 0,63 & \\
\hline
\end{tabular}

Tabl. 1. Grado de fracturación de las piezas de sílex distribuidos por niveles. / Fracturation degree of the flint pieces distributed by levels.
Al analizar la procedencia de los sílex apreciamos que se trata de un conjunto bastante homogéneo. Se han identificado seis tipos de sílex diferentes: uno mayoritario, que es el sílex de tipo Flysch con un total de 306 unidades (80,5\%); 3 tipos de sílex con una presencia minoritaria como los sílex de tipo Urbasa, Treviño y Chalosse, con un $2,6 \%, 3,7 \%$ y $2,1 \%$ respectivamente, y otros dos tipos con una presencia anecdótica como los tipos de sílex nordpirenaicos de Tercis y Salies de Béarn con una aparición del 0,8\% y 0,3\% respectivamente (Tabls. 2, 3 y 4). Finalmente en el conjunto analizado 41 ejemplares no han podido ser adjudicados a una procedencia concreta porque los criterios texturales discriminantes no se apreciaban con la suficiente claridad. Estos ejemplares se han agrupado como indeterminados y suponen el 10,0\% de la colección (Tabl. 2).

\begin{tabular}{|c|c|c|c|c|c|c|c|c|c|c|c|c|c|c|c|c|}
\hline \multirow{3}{*}{$\begin{array}{c}\text { NO } \\
\text { Retocados }\end{array}$} & \multicolumn{14}{|c|}{ TIPOS DE SÍLEX } & \multirow{2}{*}{\multicolumn{2}{|c|}{ TOTAL }} \\
\hline & \multicolumn{2}{|c|}{ Flysch } & \multicolumn{2}{|c|}{ Urbasa } & \multicolumn{2}{|c|}{ Treviño } & \multicolumn{2}{|c|}{ Chalosse } & \multicolumn{2}{|c|}{ Tercis } & \multicolumn{2}{|c|}{ Salies } & \multicolumn{2}{|c|}{ Indet. } & & \\
\hline & uds & $(\%)$ & uds & $(\%)$ & uds & $(\%)$ & uds & $(\%)$ & uds & (\%) & uds & $(\%)$ & uds & $(\%)$ & uds & (\%) \\
\hline II & 87 & 78,4 & 5 & 4,5 & 1 & 0,9 & 1 & 0,9 & 1 & 0,9 & - & - & 16 & 14,4 & 111 & 38,4 \\
\hline III & 72 & 87,8 & - & - & 6 & 7,3 & 2 & 2,4 & - & - & - & - & 2 & 2,4 & 82 & 28,4 \\
\hline IV & 66 & 84,4 & 2 & 2,6 & 1 & 1,3 & 2 & 2,6 & - & - & - & - & 7 & 9,1 & 78 & 27,0 \\
\hline $\mathrm{V}$ & 13 & 72,2 & 1 & 5,6 & 1 & 5,6 & 1 & 5,6 & - & - & - & - & 2 & 11,1 & 18 & 6,2 \\
\hline TOTAL & 238 & 82,4 & 8 & 2,8 & 9 & 3,1 & 6 & 2,1 & 1 & 0,3 & - & - & 27 & 9,3 & 289 & 100 \\
\hline \multirow{3}{*}{ Retocados } & \multicolumn{14}{|c|}{ TIPOS DE SÍLEX } & \multirow{2}{*}{\multicolumn{2}{|c|}{ TOTAL }} \\
\hline & \multicolumn{2}{|c|}{ Flysch } & \multicolumn{2}{|c|}{ Urbasa } & \multicolumn{2}{|c|}{ Treviño } & \multicolumn{2}{|c|}{ Chalosse } & \multicolumn{2}{|c|}{ Tercis } & \multicolumn{2}{|c|}{ Salies } & \multicolumn{2}{|c|}{ Indet. } & & \\
\hline & uds & $(\%)$ & uds & $(\%)$ & uds & $(\%)$ & uds & $(\%)$ & uds & $(\%)$ & uds & $(\%)$ & uds & $(\%)$ & uds & $(\%)$ \\
\hline $1+\|$ & 27 & 81,8 & 1 & 3,0 & 1 & 3,0 & - & - & 1 & 3,0 & - & - & 3 & 9,0 & 33 & 36,3 \\
\hline III & 12 & 85,7 & - & - & 1 & 7,1 & - & - & - & - & - & - & 1 & 7,4 & 14 & 15,4 \\
\hline IV & 21 & 65,6 & 1 & 3,1 & 2 & 6,3 & 2 & 6,3 & 1 & 3,1 & - & - & 5 & 15,6 & 32 & 35,2 \\
\hline V & 8 & 66,7 & - & - & 1 & 8,3 & - & - & - & - & 1 & 8,3 & 2 & 16,7 & 12 & 13,2 \\
\hline TOTAL & 68 & 74,7 & 2 & 2,2 & 5 & 5,5 & 2 & 2,2 & 2 & 2,2 & 1 & 1,1 & 11 & 12,1 & 91 & 100 \\
\hline \multirow{3}{*}{ TOTAL } & \multicolumn{14}{|c|}{ TIPOS DE SÍLEX } & \multirow{2}{*}{\multicolumn{2}{|c|}{ TOTAL }} \\
\hline & \multicolumn{2}{|c|}{ Flysch } & \multicolumn{2}{|c|}{ Urbasa } & \multicolumn{2}{|c|}{ Treviño } & \multicolumn{2}{|c|}{ Chalosse } & \multicolumn{2}{|c|}{ Tercis } & \multicolumn{2}{|c|}{ Salies } & & & & \\
\hline & uds & $(\%)$ & uds & $(\%)$ & uds & $(\%)$ & uds & $(\%)$ & uds & $(\%)$ & uds & $(\%)$ & uds & $(\%)$ & uds & $(\%)$ \\
\hline $1+\|$ & 114 & 79,2 & 6 & 4,2 & 2 & 1,4 & 1 & 0,7 & 2 & 1,4 & - & - & 19 & 13,2 & 144 & 37,9 \\
\hline III & 84 & 87,5 & - & - & 7 & 7,3 & 2 & 2,1 & - & - & - & - & 3 & 3,1 & 96 & 25,3 \\
\hline IV & 87 & 79,1 & 3 & 2,7 & 3 & 2,7 & 4 & 3,6 & 1 & 0,9 & - & - & 12 & 10,9 & 110 & 28,9 \\
\hline V & 21 & 7 & 1 & 3,3 & 2 & 6,6 & 1 & 3,3 & - & - & 1 & 3,3 & 4 & 13,3 & 30 & 7,9 \\
\hline TOTAL & 306 & 80,5 & 10 & 2,6 & 14 & 3,7 & 8 & 2,1 & 3 & 0,8 & 1 & 0,3 & 38 & 10,0 & 380 & 100 \\
\hline
\end{tabular}

Tabl. 2. Tipos de sílex clasificados por niveles. / Flint types classified by levels.

\section{2.- TIPOS DE SÍLEX}

Los cuatro tipos principales en cuanto al número de evidencias identificadas son los del Flysch (en sus variedades Kurtzia y Bidache y Gaintxurizketa), Treviño, Urbasa y Chalosse. Estos cuatro tipos son sílex trazadores para el Paleolítico Superior de la Región Cantábrica y del Pirineo occidental (TARRIÑO, 2006). También aparecen indicios de otros dos tipos de sílex como lo son los de Tercis y Salies, con únicamente la aparición de cuatro ejemplares entre ambos. En la figura 1 se puede observar el mapa de difusión de todos los sílex que han aparecido en el yacimiento.

- Sílex del Flysch. Su principal característica es la presencia de abundantes espículas de esponja. Aparecen muy patinados en el yacimiento, casi las dos terceras partes (63,8\%). Los que presentan una clara laminación turbidítica podemos sospechar con garantías que son de la variedad Bidache (algo más del 10\% de este tipo) (Fig. 3). Los afloramientos que se encuentran más cercanos al yacimiento son los de Kurtzia (Barrika, Bizkaia) (Fig. 2) 


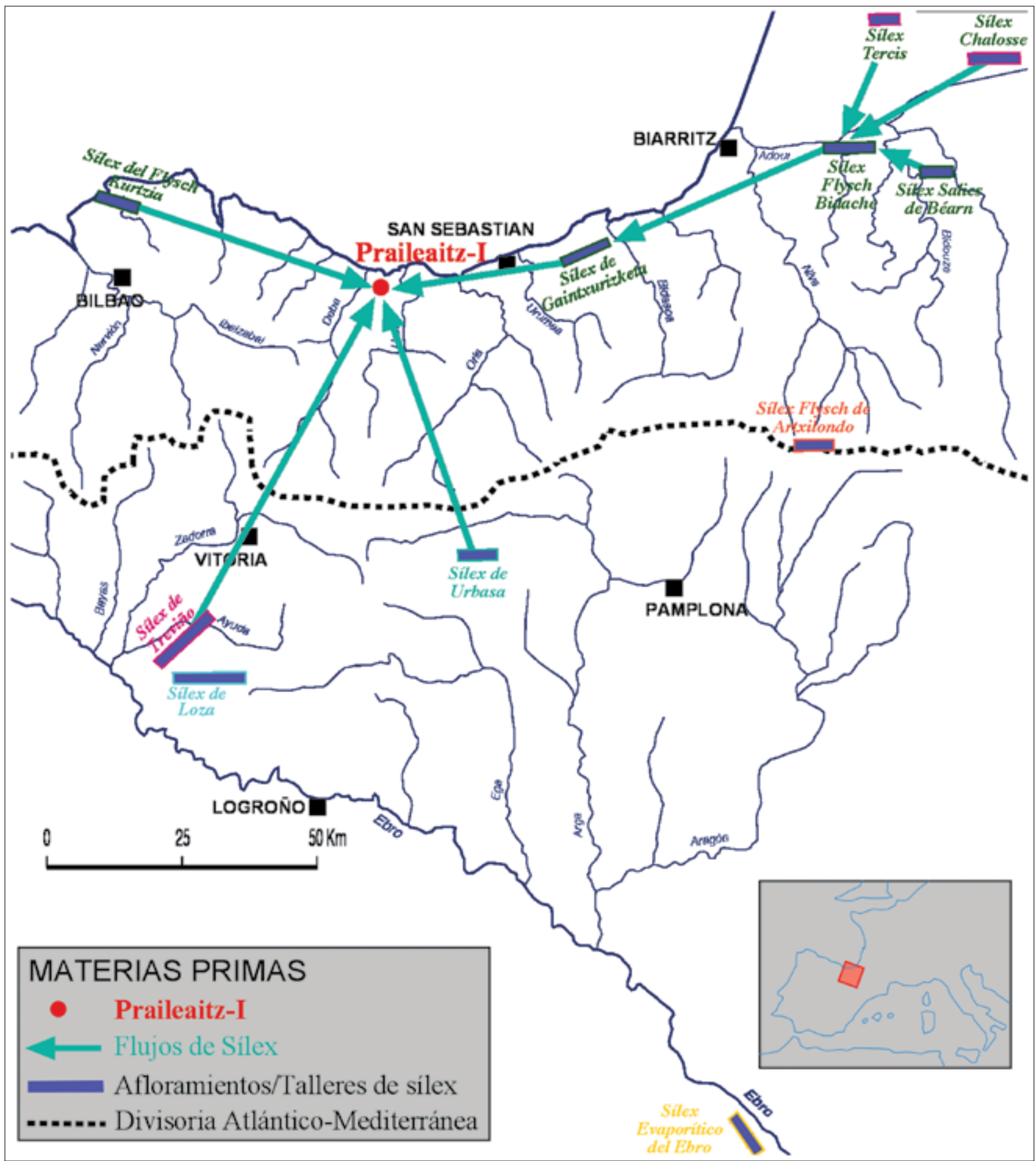

Fig. 1. Mapa de flujos de circulación de los distintos tipos de sílex desde sus afloramientos hasta el yacimiento de Praileaitz l. / Flows of the different flint types from their outcrops to the site of Praileaitz I.

a una distancia de 55-60 km. A prácticamente el doble, 100-110 km, encontramos los de Bidache (Pirineos atlánticos, Francia). También se ha podido determinar dentro de este tipo de sílex la variedad Gaintxurizketa (cinco uds.) (Fig. 4) cuyos afloramientos se encuentran al SO de Irun, a unos 50-55 km en línea recta (Fig. 1).
- Sílex de Treviño. Es el siguiente tipo en importancia en cuanto al número de restos encontrados. Son 14 fragmentos que suponen el 3,7\% del total de la colección. Sus afloramientos aparecen a $80-90 \mathrm{~km}$ en línea recta en la Sierra de Araico (Treviño, Burgos), al sur de la Cuenca Vasco Cantábrica (Fig. 1). Se han 
identificado sílex nodulares y estratiformes (silcretas) característicos de estas formaciones del Mioceno (Terciario continental) (Fig. 6).

- Sílex de Urbasa. Es el tercer tipo en abundancia con 10 evidencias (2,6\%). Aparecen muy patinados y se han observado los foraminíferos característicos de estos sílex (discocyclínidos) formados en plataformas marinas someras del Thanetiense (Paleoceno, Terciario) (Fig. 5). Sus afloramientos aparecen en la Sierra de Urbasa (Navarra) a unos 60-70 km de distancia en línea recta (Fig. 1).

- Sílex Chalosse. Se han identificado 8 fragmentos de este tipo (2,1\%). Se suelen encontrar patinados y se han identificado presencia de fósiles característicos como lepidorbitoides y briozoos. Al igual que los anteriores son sílex de plataforma, pero en este caso, del Cretácico superior (Maastrichtiense) (Fig. 7). Afloran en la región nordpirenaica de Chalosse en las inmediaciones del anticlinal de Audignon (Landas, Francia) a unos 150160 km en línea recta (Fig. 1).

- Sílex Tercis y Salies de Béarn. Se trata de sílex nordpirenaicos que aparecen de modo muy minoritario (tres y una evidencia respectivamente). Son sílex formados en ambientes marinos profundos del Cretácico superior. Sus afloramientos se sitúan a unos 130-140 km del yacimiento en línea recta (Fig. 1).

\section{3.- DATOS OBTENIDOS}

Si comparamos su aparición según sean útiles (Retocados) o productos de talla (No retocados) destaca que los del Flysch aparecen ligeramente mejor representados entre los restos de talla, ya que suponen un $82,4 \%$ $(90,8 \%$ sin contar los indeterminados) mientras que son un $74,7 \%$ (85,0\% sin contar los indeterminados) de los útiles, siendo los sílex cuyos afloramientos se encuentran más cerca del yacimiento. El comportamiento del resto de tipos minoritarios es diferente. Mientras que los de Urbasa y Chalosse se encuentran representados porcentualmente de un modo similar entre restos de talla y útiles, los de Treviño y Tercis se encuentran bastante mejor representados entre los útiles, de modo que los de Treviño prácticamente duplican su porcentaje y para los de Tercis, de los tres sílex de este tipo dos son útiles (Tabl. 2). Esta diferenciación no se aprecia entre los Indeterminados que se encuentran representados de modo similar entre los retocados y los no retocados.

De entre los sílex minoritarios destaca la aparición de los de Urbasa en los restos de talla en los niveles Epipaleolíticos (niveles I y II), con más de la mitad de ejemplares de esta procedencia (cinco de ocho uds.) que son el 4,5\% de los sílex del nivel (el 5,3\% si no se cuentan los Intederminados). También destaca la aparición de los sílex de Treviño en el nivel superior del Magdaleniense (nivel III), donde seis de los ocho sílex "no Flysch" de este nivel son de esta procedencia con un 7,3\% (el 7,5\% si no se cuentan los Indeterminados). Sin embargo, los sílex Chalosse se encuentran homogéneamente repartidos sin destacar en ninguno de los niveles.

Entre los restos de talla solo cabe destacar que los núcleos solo aparecen entre los sílex del Flysch y en muy poca cantidad (tres ejemplares) y de los ocho restos de talla de Urbasa, cinco son láminas (Tabl. 3).

Finalmente en el análisis de los útiles retocados (Tabls. 4, 5, 6, 7 y 8), solo destacar la aparición que de los sílex minoritarios es muy esporádica, solo 13 ejemplares para cinco tipos de sílex. Se encuentra muy homogéneamente repartida por los diferentes niveles. Solo destacan en el cómputo general los de Treviño que aparecen en un porcentaje del 5,4\% del total (un 6,2\% si no se cuentan los Indeterminados).

Recapitulando, se trata de una colección muy homogénea en cuanto a la procedencia (más del 90\% se trata de sílex Flysch), muy fracturada y bastante patinada. Los sílex minoritarios principales: Urbasa, Treviño y Chalosse aparecen entre un 2\% y un $4 \%$ y, anecdóticamente, se han identificados dos tipos norpirenaicos como: el sílex Tercis $(0,9 \%$, con tres ejemplares) y sílex de Salies de Béarn $(0,3 \%$, con un ejemplar).

\begin{tabular}{|c|c|c|c|c|c|c|c|c|c|}
\hline \multirow{2}{*}{ Niveles I+II } & \multicolumn{7}{|c|}{ TIPO DE SÍLEX } & \multicolumn{2}{|c|}{ TOTAL } \\
\hline & Flysch & Urbasa & Treviño & Chalosse & Tercis & Salies & Indet. & uds & $\%$ \\
\hline Lámina & 48 & 4 & & 1 & 1 & & 8 & 62 & 55,9 \\
\hline Lasca & 25 & 1 & 1 & & & & 5 & 32 & 28,8 \\
\hline Avivado & 6 & & & & & & 1 & 7 & 6,3 \\
\hline Golpe de buril & 2 & & & & & & 1 & 3 & 2,7 \\
\hline Núcleo & 3 & & & & & & & 3 & 2,7 \\
\hline Fragmento & 1 & & & & & & & 1 & 0,9 \\
\hline Microfragmento & 2 & & & & & & 1 & 3 & 2,7 \\
\hline SUBTOTAL (uds) & 87 & 5 & 1 & 1 & 1 & & 16 & 111 & 100,0 \\
\hline SUBTOTAL (\%) & 78,4 & 4,5 & 0,9 & 0,9 & 0,9 & & 14,4 & & 100,0 \\
\hline
\end{tabular}




\begin{tabular}{|c|c|c|c|c|c|c|c|c|c|}
\hline \multirow{2}{*}{ Nivel III } & \multicolumn{7}{|c|}{ TIPO DE SÍLEX } & \multicolumn{2}{|c|}{ TOTAL } \\
\hline & Flysch & Urbasa & Treviño & Chalosse & Tercis & Salies & Indet. & uds & $\%$ \\
\hline Lámina & 24 & & & 1 & & & 1 & 26 & 31,7 \\
\hline Lasca & 33 & & 4 & 1 & & & 1 & 39 & 47,6 \\
\hline \multicolumn{10}{|l|}{ Avivado } \\
\hline \multicolumn{10}{|l|}{ Golpe de buril } \\
\hline \multicolumn{10}{|l|}{ Núcleo } \\
\hline Fragmento & 1 & & 1 & & & & & 2 & 2,4 \\
\hline Microfragmento & 14 & & 1 & & & & & 15 & 18,3 \\
\hline SUBTOTAL (uds) & 72 & & 6 & 2 & & & 2 & 82 & 100,0 \\
\hline SUBTOTAL (\%) & 87,8 & & 7,3 & 2,4 & & & 2,4 & & 10 \\
\hline \multirow{2}{*}{ Nivel IV } & \multicolumn{7}{|c|}{ TIPO DE SÍLEX } & \multicolumn{2}{|c|}{ TOTAL } \\
\hline & Flysch & Urbasa & Treviño & Chalosse & Tercis & Salies & Indet. & uds & $\%$ \\
\hline Lámina & 24 & 1 & 1 & & & & 6 & 32 & 41,0 \\
\hline Lasca & 34 & 1 & & 2 & & & & 37 & 47,4 \\
\hline Avivado & 3 & & & & & & & 3 & 3,8 \\
\hline Golpe de buril & 2 & & & & & & & 2 & 2,6 \\
\hline \multicolumn{10}{|l|}{ Núcleo } \\
\hline Fragmento & 1 & & & & & & 1 & 2 & 2,6 \\
\hline Microfragmento & 2 & & & & & & & 2 & 2,6 \\
\hline SUBTOTAL (uds) & 66 & 2 & 1 & 2 & & & 7 & 78 & 100,0 \\
\hline SUBTOTAL (\%) & 84,6 & 2,6 & 1,3 & 2,6 & & & 11,6 & & 100,0 \\
\hline \multirow{2}{*}{ Nivel $\mathbf{V}$} & \multicolumn{7}{|c|}{ TIPO DE SÍLEX } & \multicolumn{2}{|c|}{ TOTAL } \\
\hline & Flysch & Urbasa & Treviño & Chalosse & Tercis & Salies & Indet. & (uds.) & $(\%)$ \\
\hline Lámina & 8 & & 1 & & & & & 9 & 5 \\
\hline Lasca & 5 & 1 & & 1 & & & 1 & 8 & 44,4 \\
\hline \multicolumn{10}{|l|}{ Avivado } \\
\hline \multicolumn{10}{|l|}{ Golpe de buril } \\
\hline \multicolumn{10}{|l|}{ Núcleo } \\
\hline Fragmento & & & & & & & 1 & 1 & 5,6 \\
\hline \multicolumn{10}{|l|}{ Microfragmento } \\
\hline SUBTOTAL (uds) & 13 & 1 & 1 & 1 & & & 2 & 18 & 100,0 \\
\hline SUBTOTAL (\%) & 72,2 & 5,6 & 5,6 & 5,6 & & & 11,1 & & 100,0 \\
\hline \multirow{2}{*}{ Todos los Niveles } & \multicolumn{7}{|c|}{ TIPO DE SÍLEX } & \multicolumn{2}{|c|}{ TOTAL } \\
\hline & Flysch & Urbasa & Treviño & Chalosse & Tercis & Salies & Indet. & (Uds.) & $(\%)$ \\
\hline Lámina & 104 & 5 & 2 & 2 & 1 & & 15 & 129 & 44,6 \\
\hline Lasca & 97 & 3 & 5 & 4 & & & 7 & 116 & 40,1 \\
\hline Avivado & 9 & & & & & & 1 & 10 & 3,5 \\
\hline Golpe de buril & 4 & & & & & & 1 & 5 & 1,7 \\
\hline Núcleo & 3 & & & & & & & 3 & 1,0 \\
\hline Fragmento & 3 & & 1 & & & & 2 & 8 & 2,1 \\
\hline Microfragmento & 18 & & 1 & & & & 1 & 22 & 6,9 \\
\hline TOTAL (uds) & 238 & 8 & 9 & 6 & 1 & & 27 & 289 & 100,0 \\
\hline TOTAL (\%) & 82,4 & 2,8 & 3,1 & 2,1 & 0,3 & & 9,3 & & 100,0 \\
\hline
\end{tabular}

Tabl. 3. Distribución de los Tipos de Restos de Talla clasificados por tipos de sílex en los diferentes niveles. / Distribution of debitage remains classified by flint types at different levels. 


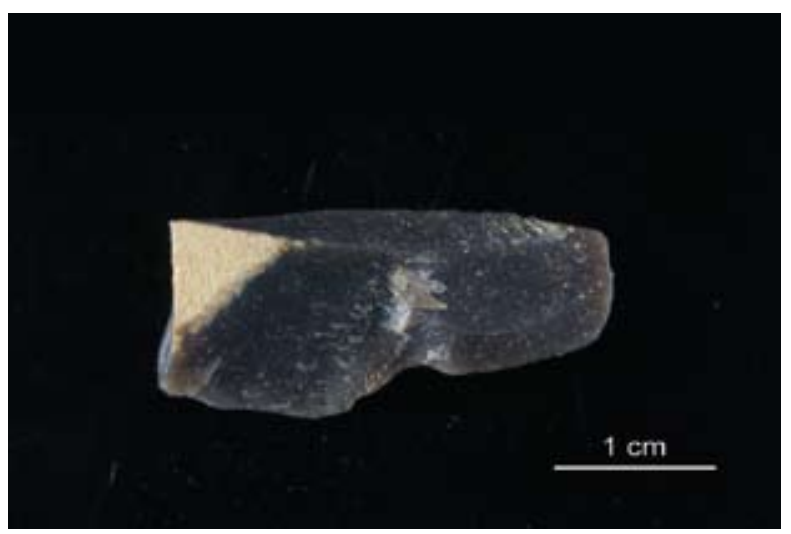

Fig. 2. Fragmento de lámina de sílex de Flysch (Kurtzia). Presenta una superficie cortical característica de esta variedad, probablemente debida a la acción marina. / Flysch flint (Kurtzia) blade. It presents a characteristic cortical surface of this variety, probably due to the marine abrasion.

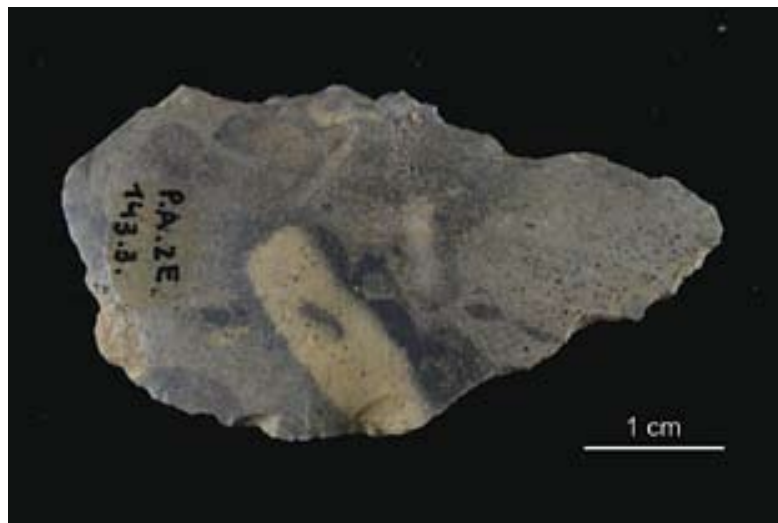

Fig. 4. Punta de sílex de tipo Flysch (Gaintxurizketa), las zonas amarillentas son bioturbaciones con mayor contenido en impurezas carbonatadas. / Flysch flint (Gaintxurizketa) projectile point, The yellowish areas are bioturbations with greater content of carbonated impurities.

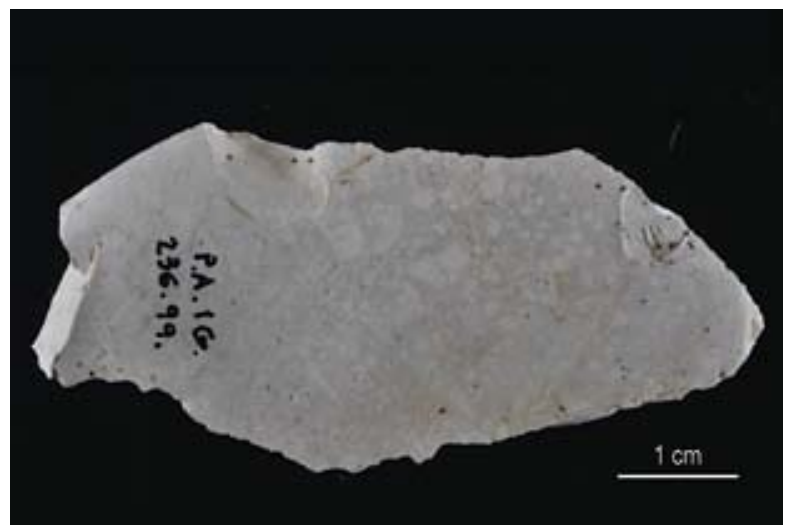

Fig. 6. Escotadura confeccionada en sílex de Treviño (silcreta brechoide). Se presenta muy patinado. / Notche made of Treviño flint (brecciated silcrete). It is very patined.

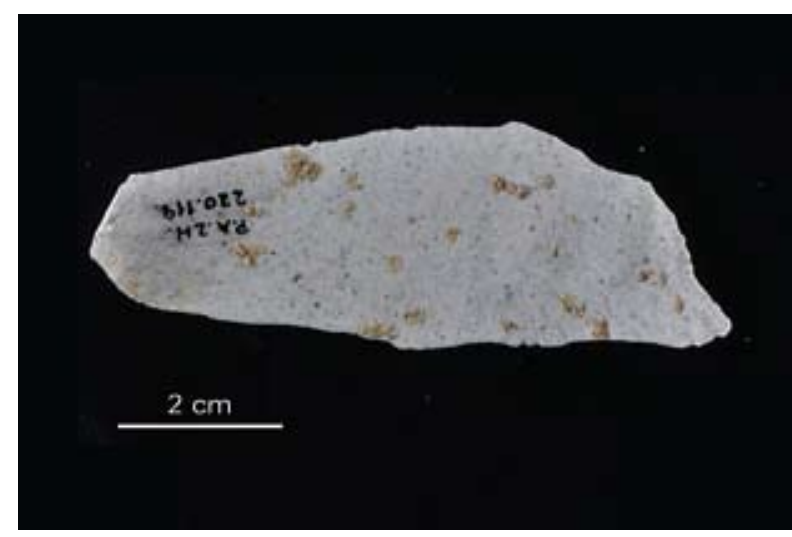

Fig. 3. Perforador confeccionado con sílex de Flysch (Bidache) En la superficie se aprecian pequeños parches de concreciones calcáreas. / Awl made of Flysch flint (Bidache). On the surface are small patches of calcareous concretions.

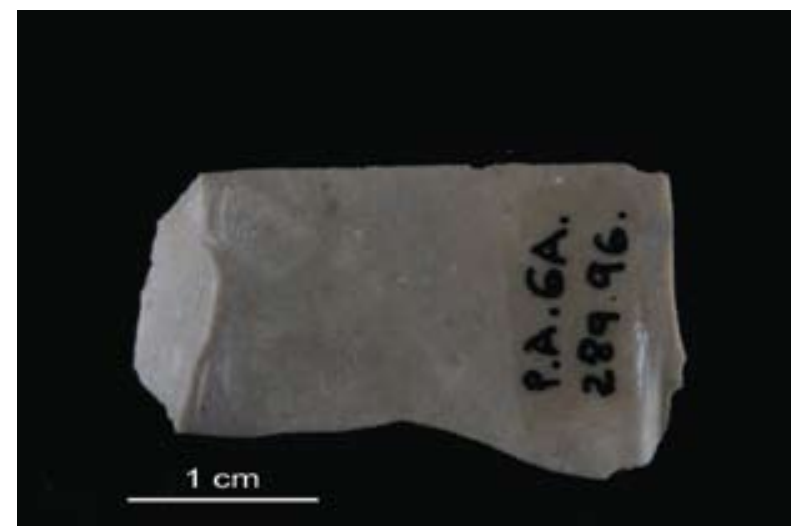

Fig. 5. Fragmento proximal de lámina confeccionado en sílex de Urbasa. / Proximal fragment of blade made of Urbasa flint.

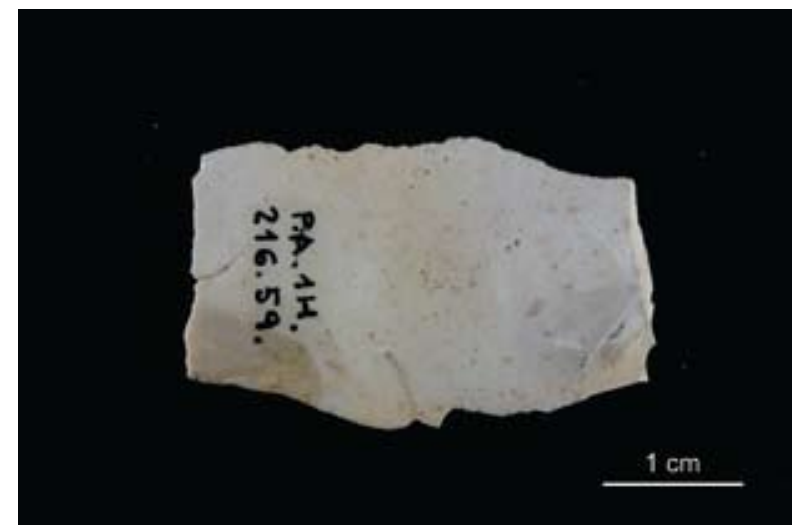

Fig. 7. Buril sobre lámina corta confeccionada en sílex de tipo Chalosse procedente de las Landas (Francia). / Burin on short blade made of Chalosse flint type from Landes (France). 
En las tablas $(4,5,6,7$ y 8$)$ se muestran los daLAPLACE, 1974) clasificados por tipos de sílex. tos obtenidos por tipos de Útiles (Grupos tipológicos de

\begin{tabular}{|c|c|c|c|c|c|c|c|c|c|c|}
\hline \multicolumn{2}{|c|}{ Retocados } & \multicolumn{7}{|c|}{ TIPO DE SÍLEX } & \multicolumn{2}{|c|}{ TOTAL } \\
\hline ORDEN & GRUPO & Flysch & Urbasa & Treviño & Chalosse & Tercis & Salies & Indet. & (Uds.) & $(\%)$ \\
\hline \multirow{3}{*}{$S$} & $\mathrm{R}$ & 2 & & & & & & & 2 & 6,1 \\
\hline & G & 4 & & & & & & & 4 & 12,1 \\
\hline & $\mathrm{D}$ & 2 & & & & & & & 2 & 6,1 \\
\hline \multirow{5}{*}{ A } & $T$ & 5 & & & & & & & 5 & 15,2 \\
\hline & $\mathrm{Bc}$ & 4 & & & & & & 1 & 5 & 15,2 \\
\hline & PD & 2 & 1 & & & & & & 3 & 9,1 \\
\hline & LD & 6 & & & & & & 2 & 8 & 24,2 \\
\hline & LDT & 1 & & & & & & & 1 & 3,0 \\
\hline B & $\mathrm{B}$ & 1 & & & & 1 & & & 2 & 6,1 \\
\hline$E$ & $E$ & & & 1 & & & & & 1 & 3,0 \\
\hline \multicolumn{2}{|c|}{ TOTAL (uds) } & 27 & 1 & 1 & & 1 & & 3 & 33 & 100,0 \\
\hline \multicolumn{2}{|l|}{ TOTAL (\%) } & 81,8 & 3,0 & 3,0 & & 3,0 & & 9,1 & & 100,0 \\
\hline \multicolumn{2}{|c|}{ TOTAL (\%) sin Indet. } & 90,0 & 3,3 & 3,3 & & 3,3 & & - & & 100,0 \\
\hline
\end{tabular}

Tabl. 4. Distribución de los Tipos de Restos Retocados clasificados por tipos de sílex en el nivel II. / Distribution of Retouched Remains types classified by flint types in level II.

\begin{tabular}{|c|c|c|c|c|c|c|c|c|c|c|}
\hline \multicolumn{2}{|c|}{ Retocados } & \multicolumn{3}{c|}{ TIPO DE SíLEX } & \multicolumn{2}{c|}{ TOTAL } \\
\hline ORDEN & GRUPO & Flysch & Urbasa & Treviño & Chalosse & Tercis & Salies & Indet. & (Uds.) & (\%) \\
\hline \multirow{3}{*}{ S } & R & 1 & & & & & & 1 & 2 & 14,3 \\
\cline { 2 - 11 } & P & 1 & & & & & & & 1 & 7,1 \\
\hline & G & 3 & & & & & & & 3 & 21,4 \\
\hline \multirow{3}{*}{ A } & T & 3 & & & & & & & 3 & 21,4 \\
\cline { 2 - 11 } & PD & 1 & & & & & & & 1 & 7,1 \\
\hline & LD & 1 & & 1 & & & & & 2 & 14,3 \\
\hline B & B & 1 & & & & & & & 1 & 7,1 \\
\hline E & E & 1 & & & & & & & 1 & 7,1 \\
\hline TOTAL (uds) & & 12 & & 1 & & & & 1 & 14 & 100,0 \\
\hline TOTAL (\%) & 85,7 & & 7,1 & & & & 7,1 & & 100,0 \\
\hline TOTAL (\%) sin Indet. & 92,3 & & 7,7 & & & & - & & 100,0 \\
\hline
\end{tabular}

Tabl. 5. Distribución de los Tipos de Restos Retocados clasificados por tipos de sílex en el nivel III. / Distribution of Retouched Remains types classified by flint types in level III.

\begin{tabular}{|c|c|c|c|c|c|c|c|c|c|c|}
\hline \multicolumn{2}{|c|}{ Retocados } & \multicolumn{7}{|c|}{ TIPO DE SÍLEX } & \multicolumn{2}{|c|}{ TOTAL } \\
\hline ORDEN & GRUPO & Flysch & Urbasa & Treviño & Chalosse & Tercis & Salies & Indet. & (Uds.) & $(\%)$ \\
\hline \multirow{3}{*}{$S$} & $\mathrm{R}$ & 3 & & & & 1 & & & 4 & 12,1 \\
\hline & $\mathrm{P}$ & 1 & & & & & & & 1 & 3,0 \\
\hline & $\mathrm{D}$ & 1 & & & & & & & 1 & 3,0 \\
\hline \multirow{4}{*}{$A$} & $\mathrm{~T}$ & & & & & & & 1 & 1 & 3,0 \\
\hline & $\mathrm{BC}$ & 3 & & & & & & & 3 & 9,1 \\
\hline & PD & 4 & 1 & & & & & 1 & 6 & 18,2 \\
\hline & LD & 7 & & 2 & 2 & & & 2 & 13 & 39,4 \\
\hline$B$ & $\mathrm{~B}$ & & & & & & & 1 & 1 & 6,1 \\
\hline$E$ & $E$ & 2 & & & & & & & 2 & 6,1 \\
\hline \multicolumn{2}{|c|}{ TOTAL (uds) } & 21 & 1 & 2 & 2 & 1 & & 5 & 32 & 100,0 \\
\hline \multicolumn{2}{|c|}{ TOTAL (\%) } & 65,6 & 3,1 & 6,3 & 6,3 & 3,1 & & 15,6 & & 100,0 \\
\hline \multicolumn{2}{|c|}{ TOTAL (\%) sin Indet. } & 77,8 & 3,7 & 7,4 & 7,4 & 3,7 & & - & & 100,0 \\
\hline
\end{tabular}

Tabl. 6. Distribución de los Tipos de Restos Retocados clasificados por tipos de sílex en el nivel IV. / Distribution of Retouched Remains types classified by flint types in level IV. 


\begin{tabular}{|c|c|c|c|c|c|c|c|c|c|c|}
\hline \multicolumn{2}{|c|}{ Retocados } & \multicolumn{7}{|c|}{ TIPO DE SÍLEX } & \multicolumn{2}{|c|}{ TOTAL } \\
\hline ORDEN & GRUPO & Flysch & Urbasa & Treviño & Chalosse & Tercis & Salies & Indet. & (Uds.) & $(\%)$ \\
\hline$S$ & $\mathrm{D}$ & 1 & & 1 & & & & 1 & 3 & 25,0 \\
\hline \multirow{4}{*}{$A$} & $\mathrm{~T}$ & 2 & & & & & & & 2 & 16,7 \\
\hline & $\mathrm{Bc}$ & 1 & & & & & & 1 & 2 & 16,7 \\
\hline & PD & 1 & & & & & & & 1 & 8,3 \\
\hline & LD & 1 & & & & & & & 1 & 8,3 \\
\hline$P$ & $\mathrm{~F}$ & 2 & & & & & & & 2 & 16,7 \\
\hline $\mathrm{B}$ & $B$ & & & & & & 1 & & 1 & 8,3 \\
\hline \multicolumn{2}{|c|}{ TOTAL (uds) } & 8 & & 1 & & & 1 & 2 & 12 & 100,0 \\
\hline \multicolumn{2}{|l|}{ TOTAL (\%) } & 66,7 & & 8,3 & & & 8,3 & 16,7 & & 100,0 \\
\hline \multicolumn{2}{|c|}{ TOTAL (\%) sin Indet } & 8 & & 1 & & & 1 & - & & 100,0 \\
\hline
\end{tabular}

Tabl. 7. Distribución de los Tipos de Restos Retocados clasificados por tipos de sílex en el nivel V. / Distribution of Retouched Remains types classified by flint types in level V.

\begin{tabular}{|c|c|c|c|c|c|c|c|c|c|c|}
\hline \multicolumn{2}{|c|}{ Retocados } & \multicolumn{7}{|c|}{ TIPO DE SÍLEX } & \multicolumn{2}{|c|}{ TOTAL } \\
\hline ORDEN & GRUPO & Flysch & Urbasa & Treviño & Chalosse & Tercis & Salies & Indet. & (Uds.) & (\%) \\
\hline \multirow{4}{*}{$S$} & $\mathrm{R}$ & 6 & & & & 1 & & 1 & 8 & 8,7 \\
\hline & $\mathrm{P}$ & 2 & & & & & & & 2 & 2,2 \\
\hline & $G$ & 7 & & & & & & & 7 & 7,6 \\
\hline & $\mathrm{D}$ & 4 & & 1 & & & & 1 & 6 & 6,5 \\
\hline \multirow{5}{*}{ A } & $T$ & 10 & & & & & & 1 & 11 & 12,0 \\
\hline & $\mathrm{BC}$ & 8 & & & & & & 2 & 10 & 10,9 \\
\hline & PD & 8 & 2 & & & & & 1 & 11 & 12,0 \\
\hline & LD & 15 & & 3 & 2 & & & 4 & 24 & 26,1 \\
\hline & LDT & 1 & & & & & & & 1 & 1,1 \\
\hline$P$ & $F$ & 2 & & & & & & & 2 & 2,2 \\
\hline B & $B$ & 2 & & & & 1 & 1 & 1 & 6 & 6,5 \\
\hline$E$ & $\mathrm{E}$ & 3 & & 1 & & & & & 4 & 4,3 \\
\hline \multicolumn{2}{|c|}{ TOTAL (uds) } & 68 & 2 & 5 & 2 & 2 & 1 & 11 & 91 & 100,0 \\
\hline \multicolumn{2}{|l|}{ TOTAL (\%) } & 74,7 & 2,2 & 5,5 & 2,2 & 2,2 & 1,1 & 12,1 & & 100,0 \\
\hline \multicolumn{2}{|c|}{ TOTAL (\%) sin Indet. } & 85,0 & 2,5 & 6,3 & 2,5 & 2,5 & 1,3 & - & & 100,0 \\
\hline
\end{tabular}

Tabl. 8. Distribución de los Tipos de Restos Retocados clasificados por tipos de sílex en el conjunto de todos los niveles. / Distribution of Retouched Remains types classified by flint types in the ensemble of all levels.

\section{4.- AGRADECIMIENTOS}

Este trabajo ha sido llevado a cabo con el apoyo de los siguientes proyectos HAR2008-05797 y RYC- 200701626 (Programa Ramón y Cajal) financiado por el Ministerio de Ciencia e Innovación (actualmente MINECO) y HAR2011-26956 del Ministerio de Economía y Competitividad (MINECO), con fondos del Fondo Social Europeo (FSE) y del Grupo Consolidado de Investigación en Prehistoria (IT-622-13) Área de Prehistoria, Dpto de Geografía, Prehistoria y Arqueología (Universidad del País Vasco, UPV/EHU).

\section{5.- BIBLIOGRAFÍA}

LAPLACE, G.

1974 La typologie analytique et structurale: Base rationnelle d'étude des industries lithiques et osseuses. Banques de données archéologiques. Colloques Nationaux du Centre National de la Recherche Scientifique. CNRS 932, 91-143.

TARRIÑO, A.

2006 El silex en la Cuenca Vasco-Cantábrica y Pirineo navarro: caracterización y su aprovechamiento en la Prehistoria. Museo Nacional y Centro de Investigación de Altamira, Monografías; 21 